\title{
Flows of Rényi entropies
}

\author{
Yu. V. Nazarov \\ Kavli Institute of NanoScience, Delft University of Technology, Lorentzweg 1, NL-2628 CJ, Delft, The Netherlands.
}

(Received 17 August 2011; revised manuscript received 31 October 2011; published 17 November 2011)

\begin{abstract}
We demonstrate that the condensed matter quantum systems encompassing two reservoirs connected by a junction permit a natural definition of flows of conserved measures, i.e., Rényi entropies. Such flows are similar to the flows of physical conserved quantities such as charge and energy. We develop a perturbation technique that permits efficient computation of Rényi entropy flows and analyze second- and fourth-order contributions. Second-order approximation was shown to correspond directly to the transition events in the system and thereby to possess a set of intuitive features. The analysis of fourth-order corrections reveals a more complicated picture: The intuitive relations do not hold anymore, and the corrections exhibit divergencies in low-temperature limit, manifesting an intriguing nonanalytical dependence of the flows on coupling strength in the limit of weak couplings and vanishing temperatures.
\end{abstract}

DOI: 10.1103/PhysRevB.84.205437

PACS number(s): 05.30.-d, 03.67.-a, 65.40.gd

\section{INTRODUCTION}

Modern condensed matter theory borrows concepts from quantum information theory, in particular, entanglement. There is an explosive growth of applications of these concepts. ${ }^{1}$ Although the measures of entanglement are not linear in density matrix and, as such, can not be directly related to quantum observables, these measures proved to provide insight into complex structure of many-body wave functions, and in many cases can be efficiently computed numerically. ${ }^{2}$ Most applications are restricted to an equilibrium situation where the object of interest is the wave function of the ground state of a system $|\Psi\rangle$. The system is cut into two subsystems $A, B$ (typically, in real space). Partial trace over a subsystem gives a reduced density matrix $\hat{R}^{(A)}=\operatorname{Tr}_{B}\{|\Psi\rangle\langle\Psi|\}$ in Hilbert space of another subsystem. Roughly, the von Neumann entropy of $\hat{R}$ can be ascribed to the boundary between the subsystems. There are a number of illuminating theoretical results ${ }^{1,3,4}$ related to the von Neumann entropy of the boundary and its scaling with the boundary area. Not only entropy is under scrutiny: the attention is paid to, and the information is gathered from, the whole spectrum of the reduced density matrix $\hat{R}^{2,5,6}$ The information about the spectrum can be compactified to a set of quantities that have been introduced in the context of information theory by Alfred Rényi ${ }^{7}$ and are called Rényi entropies. We define them as follows: ${ }^{8}$

$$
S_{M}=\operatorname{Tr}\left\{\hat{R}^{M}\right\} .
$$

Logs of $S_{M}$ are obviously extensive quantities: if $\hat{R}$ can be presented as a direct product over a number of subsystems, their contributions to $\ln S_{M}$ add. The von Neumann entropy is obtained by taking a formal limit

$$
S=-\lim _{M \rightarrow 1} \frac{\partial S_{M}}{\partial M}=-\lim _{M \rightarrow 1} \frac{\partial \ln S_{M}}{\partial M} .
$$

Several works address time evolution of entropies in the course of transition processes such as a quantum quench. ${ }^{9}$ There are interesting results concerning von Neumann entropy production in stationary nonequilibrium systems, in particular, in quantum point contacts. ${ }^{10} \mathrm{~A}$ simplest quantum point contact is a single-channel conductor with transmission coefficient $T_{0}$, which connects two electronic reservoirs. The entropy flow for zero temperature in the reservoirs was shown to be $\mathrm{e}^{10}$

$$
\frac{d S}{d t}=-\frac{d N_{\mathrm{att}}}{d t}\left[T_{0} \ln T_{0}+\left(1-T_{0}\right) \ln \left(1-T_{0}\right)\right] .
$$

Here, $\left(d N_{\text {att }} / d t\right)$ is the number of electrons per unit time that attempt to transfer the contact $\left(d N_{\text {att }} / d t\right)=e V /(2 \pi \hbar)$. The energy flow to either reservoir is given by $d E / d t=$ $\left(d N_{\text {att }} / d t\right) T_{0} e V / 2$. The approach of Ref. 10 was rather heuristic, relying on the representation of the electron many-body state in terms of a sequence of individual single-electron scattering events, ${ }^{11}$ which is instrumental in the field of full counting statistics. Levitov and Klich in Ref. 12 have rederived these results with a more microscopic approach, extended those to the case of a general time-dependent scatterer, and discovered a remarkable correspondence between the entropy flow and full counting statistics of electron transfers. This has been further elaborated in Ref. 13. References 12 and 13 reproduce Eq. (1).

There are two interesting peculiarities in the relation (1) that have not received a proper discussion so far. First, nonanalyticity of the expression (1) at small $T_{0}$ should indicate an intriguing divergence of perturbation series in $T_{0}$ that measures coupling strength between the reservoirs. Second, the second law of thermodynamics relates the entropy and heat increments for a system in thermal equilibrium at temperature $T$. From this, one would conjecture the relation between entropy and energy flows

$$
\frac{d S}{d t}=\frac{1}{k_{B} T} \frac{d E}{d t},
$$

which would seem to have a textbook status. However, the result (1) is not compatible with this conjecture. The initial motivation for the research presented in this paper was to understand these peculiarities in a general calculable framework rather than for a restrictive case of quantum point contact.

The main result of this paper is that the flows of Rényi entropies (Re-flows in short) are well defined in standard condensed matter setups comprising two reservoirs connected by a junction. Importantly, they permit a detailed evaluation in the framework of a quantum perturbation theory developed 
here. This is in contrast to the entropy flow, albeit the latter can readily be obtained by analytical continuation from integer to continuous $M$. We study the second and fourth orders of perturbation theory. The second-order results were shown to be very special: they satisfy the textbook relation (2) as well as some extra intuitive relations outlined in the text. This is because the second-order results can be put into direct correspondence with the transition events taking place in the system. Neither this correspondence nor intuitive relations hold for fourth-order corrections. These corrections exhibit nonanalytical low-temperature behavior manifesting nonanalytical dependence of the flows on coupling strength in the limit of weak couplings and vanishing temperatures.

An obvious point of criticism is that the Re-flows are unphysical since they are nonlinear in density matrix and therefore can not readily be associated with (the flows of) measurable quantities. The author shares the opinion and idea of Levitov and Klich ${ }^{12}$ that the flows of quantum information quantities may be related to the statistics of flows of quantum observables by universal relations, the general form of which is yet to be discovered. If this is true, a measurement of such statistics would in fact constitute a measurement of an unphysical flow. An alternative proposal to transfer the Rényi entropies to physical flows has been recently put forward by Cardy. ${ }^{14}$ In any case, the Re-flows characterize information exchange between the reservoirs in a rather detailed way. This makes their evaluation useful.

The structure of the paper is as follows. In Sec. II, we detail and illustrate the definition of Rényi entropies. In Sec. III, we show that Re-flows can be associated with conserving currents, these currents being rather similar to electric or energy current through a junction in quantum transport setups. Section IV is devoted to the description of a generalized Keldysh technique, which enables perturbative calculation of Re-flows in an arbitrary setup. We evaluate the flows in the second-order approximation in Sec. V. The second-order approximation is characterized by a set of specific relations, i.e., those outlined in Secs. VI and VII. In Sec. VIII, we derive the fourthorder corrections. We demonstrate that these corrections are singular in temperature in the limit of vanishing temperature (Sec. IX). For the von Neumann entropy flow, the fourth term exponentially diverges upon decreasing temperature. This indicates an interesting nonanalytical dependence of the flows in the limit of small couplings. We conclude in Sec. X.

\section{CONSERVED MEASURES}

Let us discuss the definition and straightforward properties of Rényi entropies. We start with an isolated finite quantum system, which is characterized by density matrix $\hat{R}$. We define the Rényi entropies as traces of integer powers of $\hat{R}$ :

$$
S_{M}=\operatorname{Tr}\left\{\hat{R}^{M}\right\}
$$

This definition can be easily extended to noninteger $M$,

$$
S_{M}=\operatorname{Tr}\{\exp (M \ln \hat{R})\}
$$

which identifies $S_{M}$ as Laplace transform of the spectral function of the operator $\ln \hat{R}$. This identification permits us to relate the von Neumann entropy and Rényi entropies

$$
S=-\operatorname{Tr}\{\hat{R} \ln \hat{R}\}=-\lim _{M \rightarrow 1} S_{M}=-\lim _{M \rightarrow 1} \ln S_{M} .
$$

If the system is in thermodynamic equilibrium at temperature $T$, the Rényi entropies are readily expressed in terms of the temperature-dependent free energy $F(T)$ (see, e.g., Ref. 15):

$$
\ln S_{M}=\frac{M}{k_{B} T}[F(T / M)-F(T)] .
$$

The Rényi entropies can easily be computed for simple nonequilibrium quantum systems as well. The example we will use in this paper concerns free fermions in a system of single-particle levels labeled by $k$. We ascribe the levels' arbitrary filling factors $f_{k}$. The density matrix of the system reads as

$$
\hat{R}=\prod_{k}\left[\tilde{f}_{k}\left(1-\hat{n}_{k}\right)+\hat{n}_{k} f_{k}\right],
$$

with $\hat{n}_{k} \equiv \hat{a}_{k}^{\dagger} \hat{a}_{k}$ being number operator, $\hat{a}_{k}$ being annihilation operator in the level $k$, and $\tilde{f}_{k} \equiv 1-f_{k}$. From this, the Rényi entropies read as

$$
\ln S_{M}=\sum_{k} \ln \left(\tilde{f}_{k}^{M}+f_{k}^{M}\right) .
$$

For a degenerate Fermi gas in thermal equilibrium with constant density of states $\delta_{S}^{-1}$ near the Fermi energy, this reduces to

$$
\ln S_{M}=\frac{\pi^{2} k_{B} T}{6 \delta_{S}}\left(\frac{1}{M}-M\right) .
$$

The quantum evolution of the system is governed by a Hamiltonian $\hat{H}$. The same Hamiltonian determines the evolution of the density matrix

$$
-i \hbar \frac{d \hat{R}}{d t}=[\hat{H}, \hat{R}] .
$$

Since the density matrices in different moments of time are related by unitary transform, the trace of any power of $\hat{R}$ does not depend on time.

We just proved that Rényi entropies provide a set of conserved measures for density matrix of an isolated quantum system

$$
\frac{d}{d t} S_{M}=0,
$$

in similarity to the conserved physical quantities such as energy or change. The difference is that $S_{M}$ are not linear in density matrix and therefore can not be immediately associated with any quantum observables.

While these definitions and properties may seem straightforward, there are some caveats to discuss. They are related to the traditional classical definition of entropy and its use. To illustrate, let us start with the nonequilibrium Fermi system in a pure state where $f_{k}$ are either 1 or 0 . Obviously, $S_{M}=1$ for this pure state. Let us take $\hat{H}$, which involves weak interaction between the fermions. Common knowledge suggests that the interaction causes thermalization of the fermion distribution function. After some time, the filling factors $f_{k}$ will correspond 
to a Fermi distribution at some effective temperature $T^{*}$, which depends on the initial state. It may seem that the entropies are now given by Eq. (7) and thus differ from 1. However, they must conserve! Indeed, after the Hamiltonian evolution, the system is still in a pure state.

The solution to this apparent paradox is that Eq. (6) can not be used for an interacting fermion system, however small the interaction is. We could have used an alternative definition of the entropies that is close to the original classical definition of Boltzman. Namely, we could reduce the true density matrix $\hat{R}$ to $2 \times 2$ matrices $\rho_{k}$ by taking partial trace over all fermion Fock states not involving the level $k$, take the diagonal elements of these matrices, and substitute them to (6). Such alternative definition might even seem more useful, for instance, for description of thermalization in an electron gas. However, this definition would necessarily involve an entirely subjective choice of a basis (that of noninteracting fermion Fock states) and would not give rise to conveniently conserved measures. On these grounds, we stick to quantum definition (3).

Let us now consider a bipartition of the Hilbert space $A \otimes B$ corresponding to two systems $A$ and $B$. We can now define two sets of Rényi entropies as

$$
S_{M}^{(A)}=\operatorname{Tr}_{A}\left\{\left(\hat{R}^{(A)}\right)^{M}\right\}, \quad S_{M}^{(B)}=\operatorname{Tr}_{B}\left\{\left(\hat{R}^{(B)}\right)^{M}\right\},
$$

where the reduced density matrices in two subspaces are defined with the aid of the partial traces in these subspaces:

$$
\hat{R}^{(A)}=\operatorname{Tr}_{B}\{\hat{R}\}, \quad \hat{R}^{(B)}=\operatorname{Tr}_{A}\{\hat{R}\} .
$$

If the quantum evolutions of the systems are completely independent,

$$
\hat{H}=\hat{H}_{A}+\hat{H}_{B},
$$

with $H_{A, B}$ being operators involving the corresponding subspaces only, and both sets provide the conserved measures

$$
\frac{d}{d t} S_{M}^{(A)}=\frac{d}{d t} S_{M}^{(B)}=0 .
$$

It is interesting to note that the sets of Rényi entropies are not the only conserved measures characteristic for a bipartition. Any polynomial in density matrix that is invariant with respect to the group $U_{A} \otimes U_{B}$ of unitary transforms in two subspaces would provide such a measure. To give a minimal example, let us label the states in $A(B)$ with Latin (Greek) indices. The quantity

$$
K \equiv \sum_{a, b, c ; \alpha, \beta, \gamma} R_{a \alpha, b \gamma} R_{b \beta, c \alpha} R_{c \gamma, a \beta}
$$

is a conserved measure that can be reduced neither to the Rényi entropies of the systems nor to the Rényi entropy of the whole system. The characterization of all such measures forms an interesting research task beyond the scope of this paper.

\section{FLOWS}

Let us complicate the bipartition situation by including a Hamiltonian coupling between the systems $A$ and $B$,

$$
\hat{H}=\hat{H}_{A}+\hat{H}_{B}+\hat{H}_{A B},
$$

with $\hat{H}_{A B}$ being an operator that involves degrees of freedom in both subspaces.

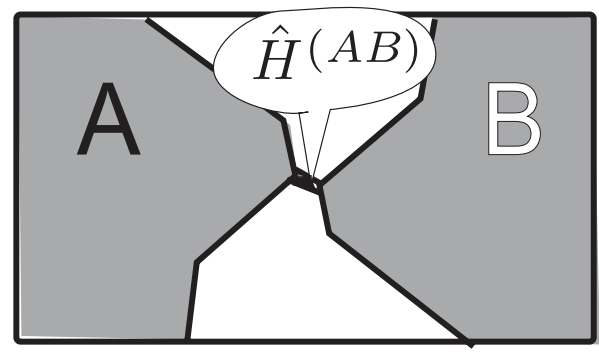

FIG. 1. Two leads (reservoirs) $A$ and $B$ in a typical quantum transport setup correspond to the bipartition $A \otimes B$. The junction connecting the two is associated with coupling Hamiltonian $H^{(A B)}$. In similarity with the flows of charge and energy, one can define the flows of Rényi entropies (Re-flows) in such setups.

We will assume that the systems $A$ and $B$ are infinitely large and are characterized by continuous excitation spectrum given by $\hat{H}_{A, B}$, respectively. However, $H_{A B}$ couples a relatively small number of degrees of freedom in both systems. It is convenient to assume that the systems $A, B$ are in thermal equilibrium at different temperatures $T_{A}, T_{B}$ and/or chemical potentials $\mu_{A}, \mu_{B}$ and, thus, play a role of reservoirs. The coupling is such that it does not lead to equilibration of $T_{A}, T_{B}$ $\left(\mu_{A}, \mu_{B}\right)$. Rather, there is a constant energy and particle flow between the systems that does not depend on actual volume of $A$ and $B$, nor on their detailed properties.

Examples of such an arrangement are common quantum transport setups. ${ }^{16}$ An exemplary setup (Fig. 1) consists of two (infinite) current-carrying leads $A$ and $B$ kept at different chemical potentials. These leads are connected by a junction. The simplest coupling that we will use in our examples is the tunneling Hamiltonian

$$
\hat{H}_{A B}=\sum_{k_{A}, k_{B}} t_{k_{A}, k_{B}}\left(\hat{a}_{k_{A}}^{\dagger} \hat{a}_{k_{B}}+\text { H.c }\right),
$$

which describes particle transfer between the leads.

It is important in quantum transport setups that the value to measure experimentally and compute theoretically is a flow of a conserved quantity, with electric charge being a simplest and most useful example. In principle, one defines a flow of charge through a cross section of the setup; this cross section may also define the bipartition. Owing to conservation, this flow does not depend on the cross section. Therefore, the exact bipartition is also not important. Owing to this, the current is said to depend on the properties of the junction rather than on details of the reservoirs. This enables the experimental investigation of nanostructures: a signal in a measurement of a nonconserving quantity would be most probably dominated by massive leads rather than by a small nanostructure, while the flow of conserved charge is determined by the nanostructure forming the junction between the leads. The same pertains to energy flow. Energy and charge are not the only conserved quantities: approximately conserved spin currents ${ }^{17}$ provide another practical example. Artificially constructed conserved quantities appear useful in description of quantum coherence in nanostructures, being the mathematical basis of the so-called circuit theory of quantum transport. ${ }^{16}$

From analogy with the flows of conserved quantities in quantum transport setups, we conjecture that there are finite 
flows of conserved measures, Rényi entropies (Re-flows), to each subsystem $A$ and $B$ :

$$
\frac{d}{d t} \ln S_{M}^{(A),(B)} \equiv \mathcal{F}_{M}^{(A)}
$$

Owing to conservation of Réneyi entropy in each system, the Re-flows would not depend on exact bipartition of the system and are determined by properties of the junction that is, in principle, described by $\hat{H}_{A B}$.

There is an important difference. For physical quantities, the conservation holds in the whole system as well as in each subsystem. For instance, electrical currents to each lead must satisfy $I_{A}+I_{B}=0 .{ }^{18}$ As far as Rényi entropies are concerned, there is no exact conservation law for a sum $\ln S_{M}^{(A)}+\ln S_{M}^{(B)}$ at finite $\hat{H}_{A B}$, although these quantities are extensive. There is a conservation law for the total Rényi entropy $\ln S^{(A+B)}$. However, the latter at finite $\hat{H}_{A B}$ is the sum $\ln S_{M}^{(A)}+\ln S_{M}^{(B)}$ only approximately, up to the terms proportional to the volume of the system.

Therefore, in general,

$$
\mathcal{F}_{M}^{(A)}+\mathcal{F}_{M}^{(B)} \neq 0
$$

Let us compute these Re-flows. We will always restrict ourselves to the Re-flow to the system $A$; the Re-flow to the system $B$ is obtained by permutation of $A$ and $B$. For brevity, we will skip the index $A$ in $S_{M}^{(A)}$ and $\mathcal{F}_{M}^{(A)}$ where this does not lead to confusion.

\section{PERTURBATION TECHNIQUE}

We will use a perturbation technique in $\hat{H}_{A B}$ and keep the calculation as general as possible. We assume adiabatic switching of the perturbation. ${ }^{19}$ Far in the past, the coupling is absent, and the density matrix is a direct product over subspaces

$$
\begin{gathered}
\hat{R}(-\infty)=\hat{R}_{A}(-\infty) \otimes \hat{R}_{B}(-\infty), \\
\hat{R}_{A}(-\infty)=\sum_{a} p_{a}|a\rangle\left\langle a\left|, \quad \hat{R}_{B}(-\infty)=\sum_{\alpha} p_{\alpha}\right| \alpha\right\rangle\langle\alpha| .
\end{gathered}
$$

[As above, we label the states in subspaces $A(B)$ with Latin (Greek) indexes.] The coupling slowly grows, achieving actual values at time $t$. The time evolution is given by (from now on, $\hbar=1$ )

$$
\begin{aligned}
\hat{R}(t)= & T \exp \left(i \int_{-\infty}^{t} d \tau \hat{H}_{A B}(\tau)\right) \hat{R}(-\infty) \\
& \times \tilde{T} \exp \left(-i \int_{-\infty}^{t} d \tau \hat{H}_{A B}(\tau)\right)
\end{aligned}
$$

where $\hat{H}_{A B}(\tau)$ is taken here in interaction representation, $T \exp (\tilde{T} \exp )$ denote time (anti)ordering in the evolution exponents. Expanding this in $H_{A B}(\tau)$ gives the perturbation series most conveniently presented as diagrams involving the Keldysh contour (Fig. 2). The operators in perturbation series are ordered along the contour. Two parts of the contour correspond to time evolution of bras and kets in the density matrix. The crosses represent the (time-dependent) perturbation $H_{A B}(t)$ at a certain time moment. The integration over time moments of all perturbations is implied. There is a

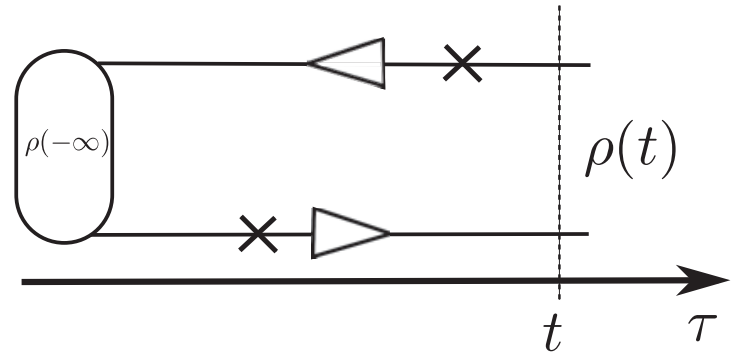

FIG. 2. Perturbation theory for a single density matrix on the Keldysh contour.

state index associated with each piece of the contour. Since $\hat{R}(-\infty)$ is diagonal, this index does not change when passing this element. The index changes if a nondiagonal matrix element of the perturbation is considered. Summation over indices is implied.

The crucial observation is that this scheme can be straightforwardly generalized to any integer number $M$ of density matrices. These matrices undergo independent unitary evolution in time interval $(-\infty, t)$. It is constructive to think of a set of $M$ parallel worlds and draw the diagrams for perturbation series using $M$ parallel bra and ket contours. To compute $S_{M}(t)$ with this set, we first need to split the contours to account for possibly different ordering of operators in subspaces $A$ and $B$ (black and white curves in Fig. 3). Then, we need to reconnect the contours at $\tau=t$. All white contours are closed within each world; this corresponds to the partial trace over $B$ for each density matrix involved. In contrast to this, the black contours are connected to form a single loop going through all the worlds; this corresponds to the matrix multiplication in the definition (3) of Rényi entropy. This conveniently represents

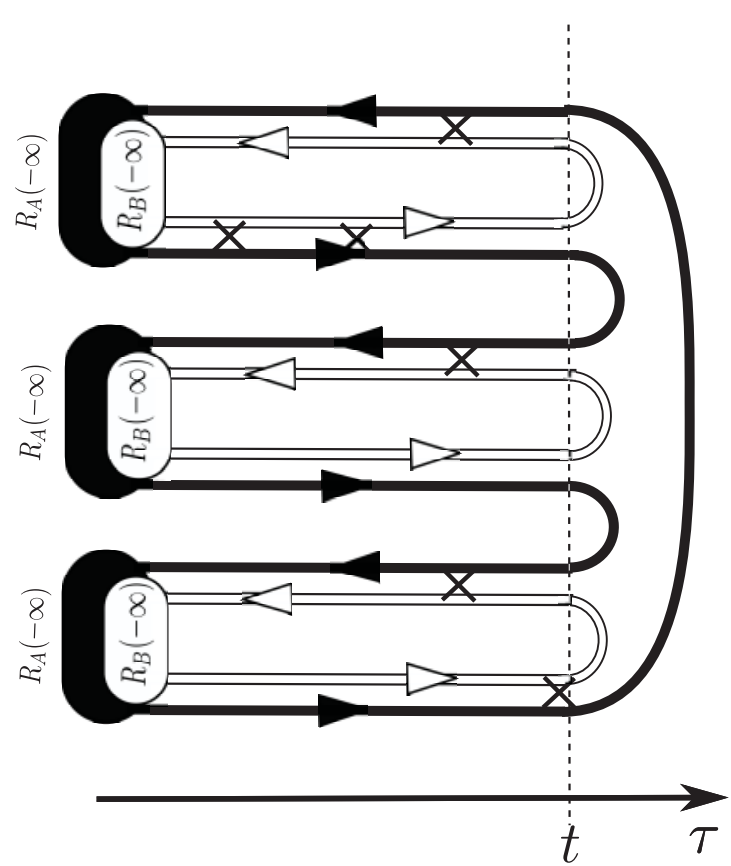

FIG. 3. A diagram of perturbation theory for $S_{M}^{(A)}$ for $M=3$. It involves three parallel worlds. Reconnection of Keldysh contours for subspaces $A$ (black) and $B$ (white) accounts for partial trace over $B$ and matrix multiplication in $A$. 
the rules of operator ordering for any diagram of particular order in $H_{A B}$.

It is interesting to note that reconnecting the contours in a different fashion gives rise to perturbation theory for other conserved measures, for instance, for $K$ given by Eq. (10). It is also interesting to see a connection between the approach suggested here and the proposal of Cardy ${ }^{14}$ to measure Rényi entropies with the aid of a quantum quench. The idea of the proposal is as follows. Let us consider a system with bipartition $A \otimes B$. Let us take $M$ identical copies of the system in its ground state, numbered by $j$. At the time moment $t=t_{0}$, the sudden quantum quench happens: it amounts to reconnecting the partitions in such a way that $A_{j}$ is connected to $B_{j+1}$ at $t>t_{0}$ (if $j=M, j+1=1$ ). After such an intrusion, there is still a chance that the system remains in the ground state: it happens to be given by $\mathcal{S}_{M} \cdot{ }^{14}$ The proposal in fact aims to physical realization of the outlined multiple-contour scheme. The parallel worlds are the copies of the system, and a certain type of contour reconnection is realized with the quantum quench described.

It is natural to require that the matrix elements of $H_{A B}$ are only nondiagonal, that is, $H_{a \alpha, b \beta}^{(A B)}=0$ if either $a=b$ or $\alpha=\beta$. In this case, the first nonvanishing contributions to Re-flows will be of the second order in $H_{A B}$.

\section{SECOND ORDER}

Let us compute the Re-flows in the second order in $H_{A B}$. It is proficient to directly compute the time derivative of $S_{M}$. For diagrams, this corresponds to placing one of the perturbations at $\tau=t$. The only way to satisfy the continuity of state index along the white contours is to place the second perturbation in the same world. Four contributing diagrams are given in Fig. 4. In fact, the same four diagrams arise in the derivation of the golden rule transition rate and are familiar to anyone who made use of the Keldysh perturbation theory for the density matrix. The specifics of Rényi entropies is reflected in the extra factors $p_{a}^{M-1}$ that the diagrams acquire in comparison with the case of a single density matrix. Summing up the four diagrams yields

$$
\begin{aligned}
\frac{\partial}{\partial t} S_{M}= & \left(-M \sum_{a, \alpha ; b, \beta}\left|H_{a \alpha, b \beta}^{(A B)}\right|^{2} p_{a}^{M} p_{\alpha}\right. \\
& \left.+M \sum_{a, \alpha ; b, \beta}\left|H_{a \alpha, b \beta}^{(A B)}\right|^{2} p_{b} p_{\beta} p_{a}^{M-1}\right) \\
& \times \int_{-\infty}^{t} d t^{\prime} 2 \operatorname{Re}\left(e^{i\left(t-t^{\prime}\right)\left(E_{i}+E_{\alpha}-E_{j}-E_{\beta}\right)}\right) .
\end{aligned}
$$

The integral over time $t^{\prime}$ reduces to

$$
2 \pi \delta\left(E_{a}+E_{\alpha}-E_{b}-E_{\beta}\right),
$$

manifesting energy conservation between the initial state $|a \alpha\rangle$ and final state $|b \beta\rangle$.

This suggests that we can rewrite the whole expression in terms of golden rule rates $\Gamma_{a \alpha, b \beta}$ of the transitions between the states $|a \alpha\rangle$ and $|b \beta\rangle$ :

$$
\Gamma_{a \alpha, b \beta}=2 \pi\left|H_{a \alpha, b \beta}^{(A B)}\right|^{2} \delta\left(E_{a}+E_{\alpha}-E_{b}-E_{\beta}\right) .
$$
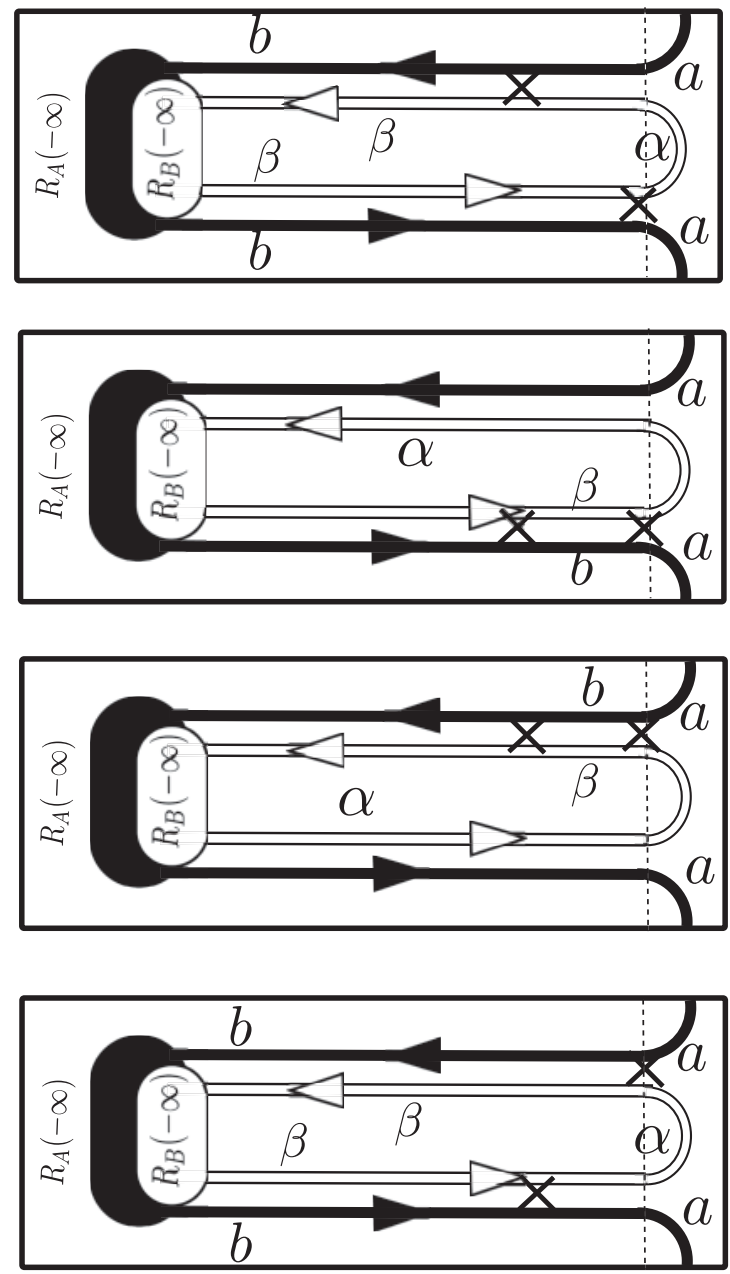

FIG. 4. Second-order diagrams for time derivative of a Rényi entropy. The contributions come only from perturbations $\hat{H}^{(A B)}$ in the same world; only this world is shown in each diagram. For all diagrams, the perturbations are taken at time moments $t$ and $t^{\prime}<t$. The letters at the contours label the states involved.

With this, the flow reads as

$$
\left(S_{M}\right) \mathcal{F}_{M}=M \sum_{a, \alpha ; b, \beta} \Gamma_{a \alpha ; b \beta}\left(p_{b} p_{\beta}-p_{a} p_{\alpha}\right) p_{a}^{M-1} .
$$

We see that the flow vanishes if the systems are in thermodynamic equilibrium at the same temperature. Indeed, in this case, $p_{b} p_{\beta} / p_{a} p_{\alpha}=\exp \left[\left(E_{b}+E_{\beta}-E_{a}-E_{\alpha}\right) / k_{B} T\right]=1$.

Since the transition rates $\Gamma_{a \alpha, b \beta}$ in the golden rule approximation are symmetric with respect to a permutation $a \alpha \leftrightarrow b \beta$, we can regroup the terms to arrive at

$$
\left(S_{M}\right) \mathcal{F}_{M}=M \sum_{a, b} \Gamma_{a \rightarrow b} p_{a}\left(p_{b}^{M-1}-p_{a}^{M-1}\right),
$$

where

$$
\Gamma_{a \rightarrow b}=\sum_{\alpha, \beta} \Gamma_{a \alpha ; b \beta} p_{\alpha}
$$

gives the total transition rate from the state $|a\rangle$ to the state $|b\rangle$ averaged over all possible configurations of system $B$. Let us use Eq. (16) to derive a simplified expression valid in zero-temperature limit. In this limit, the system $A$ is initially 
in the ground state $|0\rangle$, so that $p_{0}=1$ and $p_{a}=0$ for $a \neq 0$, $S_{M}=1$. We obtain

$$
\mathcal{F}_{M}=-M \Gamma_{0}
$$

with $\Gamma_{0}$ being the total transition rate from the ground state to any other state. Remarkably, this involves no assumption concerning the system $B$ : it can be very far from equilibrium.

Equation (16) is also a convenient starting point to derive the expression for the von Neumann entropy flow. Taking the limit $M \rightarrow 1$, we obtain

$$
-\frac{\partial S}{\partial t}=\sum_{a, b} \ln \left(p_{b} / p_{a}\right) \Gamma_{a \rightarrow b} p_{a} .
$$

Let us assume thermal equilibrium of $A$. In this case, $\ln \left(p_{b} / p_{a}\right)=\left[\left(E_{a}-E_{b}\right) / k_{B} T\right]$. Summing up the energy changes $E_{b}-E_{a}$ in the course of individual transitions from $a$ to $b$, we prove that the energy flow to the system $A$ equals

$$
\frac{d E}{d t}=\sum_{a, b} \Gamma_{a \rightarrow b}\left(E_{b}-E_{a}\right) p_{a} .
$$

Comparing this with Eq. (18), we recover the relation (2), which appears to be universally valid within the second-order perturbation theory. Remarkably, this involves no assumption about the system $B$.

One can easily imagine the situation where $d E / d t$ remains constant at $T \rightarrow 0$ [see Eq. (1)]. The entropy flow in this case diverges as $1 / T$. One can wonder how it is compatible with the fact that Re-flows approach finite limit at vanishing temperature. The point is a hidden nonanalyticity of (17): the flow does not vanish in the limit $M \rightarrow 1$ as it is implied by more general Eq. (16). Therefore, Eq. (17) can not be immediately used to derive the flow of von Neumann entropy; rather, a general formula valid for small but finite $T$ is required for this purpose. One can see it as noncommunicativity of the limits of vanishing coupling $H_{A B} \rightarrow 0$ and vanishing temperature $T \rightarrow 0$.

\section{EXAMPLE: FERMI GAS}

Let us explicitly compute the Re-flows for tunneling between two nonequilibrium Fermi gases. In this case, $a$ (or $b$ ) is a number state defined by a set of occupation numbers in all levels $k\left\{n_{k}\right\}, p_{a}=\prod_{k}\left[\tilde{f}_{k}\left(1-n_{k}\right)+f_{k}\left(n_{k}\right)\right]$. The perturbation $\hat{H}_{A B}$ is given by Eq. (12). Let us concentrate on the transitions involving a level $q$. Since the probabilities factorize, we can forget about the Fock states involving all other levels since their contributions to the right-hand side of Eq. (16) cancel with their contribution to $S_{M}$. We can therefore consider only two states spanned by level $q$, substituting into Eq. (16) their contribution to $S_{M}=f_{q}^{M}+\tilde{f}_{q}^{M}$. The probabilities of these states $|0\rangle\left(n_{q}=0\right)$ and $|1\rangle\left(n_{q}=1\right)$ are $f_{q}$ and $\tilde{f}_{q}$, respectively. Substituting this into Eq. (16) and summing up over $q$ yields

$$
\mathcal{F}_{M}=M \sum_{q}\left(\Gamma_{q}^{+} \tilde{f}_{q}-\Gamma_{q}^{-} f_{q}\right) \frac{f_{q}^{M-1}-\tilde{f}_{q}^{M-1}}{f_{q}^{M}+\tilde{f}_{q}^{M}},
$$

with $\Gamma_{q}^{+}\left(\Gamma_{q}^{-}\right)$being the rates of addition (extraction) of a fermion to (from) the level $q$.
Let us outline a heuristic way to obtain the above relation. This way does not involve any quantum mechanics: it is something Boltzmann would do if given the problem. Let us treat $\ln S_{M}$ and $f_{q}$ as continuous quantities related by Eq. (6). By virtue of the relation, the flux of $\ln S_{M}$ would be expressed in terms of fluxes of $f_{q}$ as

$$
\frac{d}{d t} \ln S_{M}=\sum_{q} \frac{\partial \ln S_{M}}{\partial f_{k}}\left(\frac{d f_{k}}{d t}\right) .
$$

Since $f_{q}$ is the average number of particles in the level $q$,

$$
\frac{d f_{q}}{d t}=\Gamma_{q}^{+} \tilde{f}_{q}-\Gamma_{q}^{-} f_{q} .
$$

Combining these two relations reproduces Eq. (19)! We stress that we do not see any reason for this relation to hold, in general, since both $S_{M}$ and $n_{q}$ change in discrete rather than continuous fashion in the course of a tunneling transition. Indeed, the inspection of fourth-order terms in perturbation theory shows that the heuristic way does not generally work.

Let us assume that the filling factors in the system $A$ depend on single-particle energy $E$ only. The Eq. (20) reduces to

$$
\mathcal{F}_{M}=M \int d E\left(\Gamma^{+} \tilde{f}-\Gamma^{-} f\right) \frac{f^{M-1}-\tilde{f}^{M-1}}{f^{M}+\tilde{f}^{M}},
$$

with $\Gamma^{+,-}(E)$ being addition (extraction) rates per energy interval. In case of vanishing temperature (in the system $A$ ), $f_{q}=\Theta(-E+\mu)$. The Rényi entropy flow reduces to

$$
\begin{aligned}
\mathcal{F}_{M}= & M \int d E\left[\Gamma^{+}(E) \Theta(E-\mu)\right. \\
& \left.-\Gamma^{-}(E) \Theta(-E+\mu)\right] \operatorname{sgn}(E-\mu) \\
= & -M \frac{d}{d t}\left(N^{+}+N^{-}\right)
\end{aligned}
$$

with $N^{ \pm}$being numbers of added (extracted) particles. This is in agreement with the general relation (17) since the only events that happen in our setup are indeed additions or extractions of single particles. As in the general case, analytical continuation to $M \rightarrow 1$ at vanishing temperature does not work since the expression does not vanish in the limit $M \rightarrow 1$. One can work at nonzero temperature, where it does vanish. Taking the derivative of Eq. (21) at $M \rightarrow 1$ yields the flow of von Neumann entropy

$$
\frac{\partial S}{\partial t}=\int d E\left[\Gamma^{+}(E) \tilde{f}(E)-\Gamma^{-}(E) f(E)\right] \ln \left(\frac{f}{\tilde{f}}\right) .
$$

Substituting Fermi distribution, we recover once again the relation (2) between the entropy and energy flows.

\section{MASTER-EQUATION APPROACH}

In a single world, there is a simple way to resum the perturbation series and arrive to a master equation that contains only diagonal elements of density matrix (Fig. 5). For a diagram, we split the timeline by perturbations into the blocks as shown in the figure. The blocks come in two sorts: diagonal with the same state index in both contours and nondiagonal ones. Integration over time duration of nondiagonal blocks gives golden rule transition rates. The integration over time duration of diagonal blocks is divergent; this indicates that 


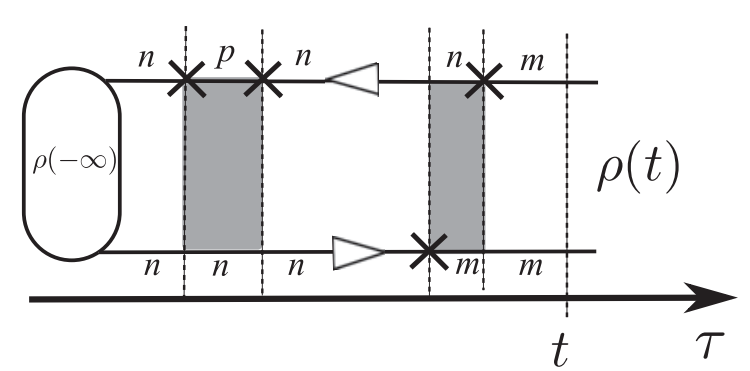

FIG. 5. Master equation in a single world is obtained from the resummation of perturbation series whereby the timeline is separated into diagonal and nondiagonal (gray shaded) blocks. One can do this resummation in multiple worlds to compute the time evolution of Rényi entropies.

the diagrams need to be resummed. The resummation gives the master equation involving the diagonal elements of the density matrix only. In the case of bipartition, this equation reads as

$$
\frac{d}{d t} p_{a \alpha}=-\Gamma_{a \alpha} p_{a \alpha}+\Gamma_{a \alpha, b \beta} p_{b \beta} .
$$

Here, $\Gamma_{a \alpha}$ stands for the total transition rate from the state $|a \alpha\rangle$, that is, the sum of the partial transition rates $\Gamma_{a \alpha}=\sum_{b \beta} \Gamma_{a \alpha, b \beta}$.

Inspired by the above exercises with second-order diagrams, one can attempt the resummation in parallel worlds in such a way that the perturbations in a nondiagonal block are situated in the same world. In this case, we have independent master equations governing the dynamics of density matrix in each world.

Suppose we know the propagator of the master equation, that is, a linear kernel $\mathcal{P}$ expressing the evolution of the probabilities:

$$
p_{a \alpha}(t)=\mathcal{P}_{a \alpha, b \beta}\left(t, t^{\prime}\right) p_{b \beta}\left(t^{\prime}\right) .
$$

With this, we can easily express time evolution of $S_{M}$ in time interval $(0, t)$ :

$$
S_{M}(t)=\sum_{a}\left(\sum_{\alpha} \mathcal{P}_{a \alpha, b \beta}(t, 0) p_{b}(0) p_{\beta}(0)\right)^{M}
$$

and compute the Rényi entropy flows. We will not analyze the general situation here since, as all systems described by a master equation, it belongs to the realm of classical physics anyway. Rather, we concentrate on a simple but constructive example. We will assume that initially the systems were in a pure product state. Let us denote this state $|00\rangle$. Let us further assume that the transitions from this state as well from all other state can happen to a great number of states. This number is so big that, once the first transition has taken place, the system will never get back to the original state. With this, the probability to remain in the state $|00\rangle$ falls off exponentially:

$$
p_{00}=\exp \left(-\Gamma_{00} t\right),
$$

with $\Gamma_{00}$ being the total rate of all transitions from $|00\rangle$. Under the same conditions, the sum in $S_{M}$ is dominated by the probability to remain in the state $|00\rangle$. The $S_{M}(t)$ just equals the probability that, during this time interval, no transition event occurs in any of $M$ parallel worlds:

$$
S_{M}(t)=p_{00}^{M}=\exp \left(-M \Gamma_{00} t\right)
$$

The flow is, therefore, $\mathcal{F}_{M}=-M \Gamma_{00}$, in full correspondence with Eq. (17).

We note that the resummation of the diagrams we have done is not complete since we have assumed that each nondiagonal block comprises only two perturbations. The transition rates are thus approximated by their golden rule expressions. In most cases involving a master equation in a single world, this is not important: the further orders of perturbation theory provide small corrections to the rates not changing the structure of the equation. It looks like this is not so in the case of parallel worlds. The analysis of fourth-order perturbation corrections presented below indicates the problems involved.

\section{FOURTH ORDER}

Let us analyze the fourth-order diagrams for the time derivative of $S_{M}$. As above, we assume that $H_{A B}$ does not contain diagonal elements. Since white contours are closed within each world, the four perturbations can either all come in the same world or in two pairs in two different worlds. If all four come in the same world, they describe a correction to one of the golden rule transition rates. This correction is to a coefficient in a second-order expression and therefore does not bring anything new. Thus motivated, we disregard these diagrams in further consideration.

An example diagram involving two different worlds is given in Fig. 6. It is important to note that, in general, the black contour entering a world with perturbations exits it with a different state index $a \neq b$. This is in contrast to the second-order diagrams. For a particular case wherein these indices are the same, $a=b$, the fourth-order diagram diverges upon integration over time. This is not surprising. By employing the perturbation technique, we expand the exponent of a Re-flow $S_{M}(t) \propto \exp \left(\mathcal{F}_{M} t\right)$ rather than the Re-flow $\mathcal{F}_{M}$. The fourth-order expansion we deal with should thus contain the terms $\propto\left(\mathcal{F}_{M}^{(2)}\right)^{2} t / 2$, with $\mathcal{F}^{(2)}$ being the second-order contribution to the rate that we have already calculated. Indeed,

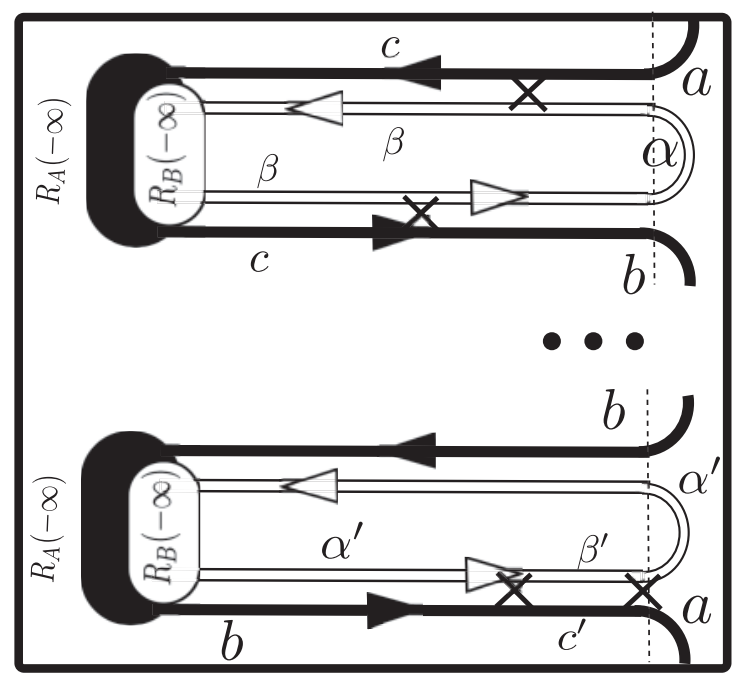

FIG. 6. A fourth-order quantum diagram for Rényi entropy flows. The contributions come from perturbations $\hat{H}^{(A B)}$ in two different worlds; only these two worlds are shown. The letters on the contours label the states involved. 
the inspection of the diagrams with $a=b$ proves that they provide the contribution expected [proportional to $\left(\mathcal{F}^{(2)}\right)^{2}$ ]. Therefore, the diagrams with $a=b$ do not contribute to the fourth-order correction to the flow, and their unpleasant divergency can be safely disregarded.

We, thus, concentrate on the case $a \neq b$. We dub these diagrams quantum: We will see that they do not permit an interpretation in terms of classical transition events. To keep notations short, all expressions for $\mathcal{F}, d S / d t$ further in this section give the fourth-order corrections to the corresponding Re-flows or entropy flow rather than the full quantities.

There are 16 diagrams of this sort to consider (only one is shown in Fig. 6). Their number corresponds to the number of ways the pairs of $\hat{H}^{(A B)}$ in each world can be placed on bra and ket contours. We need to sum up all of them intelligently, so we can present the fourth-order correction in the following form:

$$
\begin{aligned}
\frac{d}{d t} S_{M}^{(A)}=\pi \sum_{a, b}\left|A_{a b}\right|^{2} \delta\left(E_{a}-E_{b}\right) \frac{p_{a}^{M-1}-p_{b}^{M-1}}{p_{a}-p_{b}} \\
A_{a b}=\sum_{c, \alpha, \beta} H_{a \alpha, c \beta}^{(A B)} H_{c \beta, b \alpha}^{(A B)} \\
\quad \times\left(\pi\left[\left(p_{a}+p_{b}\right) p_{\alpha}-2 p_{c} p_{\beta}\right] \delta\left(E_{a}+E_{\alpha}-E_{c}-E_{\beta}\right)\right. \\
\left.\quad-i \frac{p_{a}-p_{b}}{E_{a}+E_{\alpha}-E_{c}-E_{\beta}}\right) .
\end{aligned}
$$

Let us try to comprehend the structure of the result and compare it to familiar expressions of the usual quantum perturbation theory not involving multiple worlds. The expression reminds of a golden rule formula: It contains the square of the amplitude, the delta-function of energy, which in the golden rule case would guarantee energy conservation in the course of transition between the initial and ground states, and an expression involving probabilities. To support the golden rule interpretation, the structure of the matrix elements in the amplitude $A_{a b}$ is the same as for a second-order amplitude of the transition from the state $|a \alpha\rangle$ to the state $|b \alpha\rangle$, that is, without the change of the state of the subsystem $B$. Such a transition would proceed via an intermediate virtual state $|c, \beta\rangle$. However, the further analysis of the expression does not support this interpretation. Rather, the probabilities $p_{a}, p_{b}, p_{\alpha}, p_{\beta}$ enter in a form suggesting that the transition takes place between one of the states $|a \alpha\rangle,|b \alpha\rangle$, and the state $|c \beta\rangle$. Therefore, the expression (25) can be associated with no classical transition and corresponds to no actual transition rate. Its unconventional structure arises from the fact that we address an unphysical quantum-informational quantity. This example is important from a conceptual point of view since it illustrates that quantum dynamics in parallel worlds can not generally be described in terms of transitions: the validity of such a description (Sec. VII) is restricted to the second order.

To proceed further, let us assume that the probabilities in the system $A$ depend only on energies of the corresponding states (this assumption is less restrictive than the assumption of thermal equilibrium). Then, it follows from $E_{a}=E_{b}$ that $p_{a}=p_{b}$. The term in $A_{a b}$ with the energy difference in the denominator vanishes and the expression for the fourth-order correction to a Re-flow reduces to

$$
\begin{aligned}
S_{M} \mathcal{F}_{M}= & (M-1) \pi \sum_{a, b}\left|A_{a b}\right|^{2} \delta\left(E_{a}-E_{b}\right) p_{a}^{M-2}, \\
A_{a b}= & 2 \pi \sum_{c, \alpha, \beta} H_{a \alpha, c \beta}^{(A B)} H_{c \beta, b \alpha}^{(A B)} \\
& \times\left(p_{a} p_{\alpha}-p_{c} p_{\beta}\right) \delta\left(E_{a}+E_{\alpha}-E_{c}-E_{\beta}\right) .
\end{aligned}
$$

We notice now that if the both systems are in thermal equilibrium at the same temperature, it follows from $E_{a}+$ $E_{\alpha}=E_{c}+E_{\beta}$ that $p_{a} p_{\alpha}-p_{c} p_{\beta}$ and the amplitudes $A_{a b}$ vanish. This explicitly proves the absence of Re-flows in thermal equilibrium.

Let us compute the fourth-order contribution for our favorite example of nonequilibrium Fermi gas. We pick up two levels in the system $A, k_{1,2}$, with $E_{k_{1}}=E_{k_{2}}=E$. Their contribution to $S_{M}$ reads as $S_{M}=\left[f_{A}(E)+\tilde{f}_{A}(E)\right]^{2}$. Let the state $a$ correspond to $|1\rangle_{k_{1}}|0\rangle_{k_{2}}$ and state $b$ correspond to $|0\rangle_{k_{1}}|1\rangle_{k_{2}}$ : the probabilities of these states equal $f_{A}(E) \tilde{f}_{A}(E)$. The amplitude $A_{a b}$ is contributed by all levels $q$ in the system $B$ with the energy $E_{q}=E$. To simplify further, we notice that there are two possible virtual states $|c \beta\rangle$. The first possibility is an empty level $q$ and both levels $k_{1,2}$ occupied [ $p \alpha=$ $\left.f_{B}(E), p_{\beta}=\tilde{f}_{B}(E), p_{c}=f_{A}^{2}(E)\right]$. The second possibility is an occupied level $q$ and empty levels $k_{1,2}\left[p \alpha=\tilde{f}_{B}(E)\right.$, $\left.p_{\beta}=f_{B}(E), p_{c}=\tilde{f}_{A}^{2}(E)\right]$. Owing to anticommutativity of fermionic creation and annihilation operators, these two contributions to the amplitude come with opposite sign, yielding

$$
A_{a b}=2 \pi\left[f_{B}(E)-f_{A}(E)\right] \sum_{q} t_{k_{1}, q} t_{k_{2}, q} \delta\left(E_{q}-E\right) .
$$

To get the contribution of the two levels $k_{1,2}$ to the Re-flow, we square $A_{a b}$, multiply it with $p_{\alpha}^{M-2}=\left(f_{A} \tilde{f}_{A}\right)^{M_{2}}$, and divide with the contribution $S_{M}=\left(f_{A}^{M}+\tilde{f}_{A}^{M}\right)^{2}$ of these levels to Rényi entropy. At the next step, we sum over all states in an energy interval $(E, E+d E)$ and integrate over the energy $E$ to obtain the total Re-flow in terms of an integral over energy rather then a sum over discrete levels. We obtain

$$
\mathcal{F}_{M}=(1-M) \int d E\left(f_{B}-f_{A}\right)^{2} \frac{\left(f_{A} \tilde{f}_{A}\right)^{M-2}}{\left(f_{A}^{M}+\tilde{f}_{A}^{M}\right)^{2}} \Xi(E),
$$

where $\Xi(E)$ is defined as

$$
\begin{aligned}
\Xi(E)= & 8 \pi^{2} \sum_{k, k^{\prime}, q, q^{\prime}} t_{k, q} t_{k^{\prime}, q^{\prime}} t_{k^{\prime}, q} t_{k, q^{\prime}} \\
& \times \delta\left(E-E_{k}\right) \delta\left(E-E_{k}^{\prime}\right) \delta\left(E-E_{q}\right) \delta\left(E-E_{q}^{\prime}\right) .
\end{aligned}
$$

Since the quantity $\Xi(E)$ is contributed by many discrete levels, its energy dependence is smooth in the limit of an infinite system.

\section{LOW-TEMPERATURE SINGULARITIES}

We will now explicitly demonstrate that the quantum contribution (28) derived manifests serious problems with the term-by-term perturbation theory in the limit of vanishing temperature. This indicates a nonanalytical dependence of the flows on the coupling strength in the limit of weak couplings and vanishing temperatures. 
First, let us note that the contribution seems to have an evident zero-temperature limit, namely, zero, at least if the ground state of the system $A$ is not degenerate. Indeed, in the latter case, the delta function in Eq. (25) can not be satisfied for any state $b \neq a$, and the contribution vanishes if the system is in a unique ground state, that is, at zero temperature. However, the analytical continuation to noninteger $M$ gives rise to problems: the result of the continuation at finite temperature does not have to vanish in the limit $T \rightarrow 0$. Let us illustrate this by computing (28) while assuming the thermal distribution and the difference between chemical potentials in $A$ and $B, e V$, to be much bigger than $k_{B} T$. To this end, we can set $f_{B}=0$ in Eq. (28). By disregarding the energy dependence of $\Xi$ near the Fermi surface, we obtain

$$
\mathcal{F}_{M}=k_{B} T \Xi \frac{1-M}{M}\left[1+\frac{2 \pi}{M}\left(\frac{1}{\sin \frac{\pi}{M}}-\frac{1}{\sin \frac{2 \pi}{M}}\right)\right] .
$$

While the flow vanishes at vanishing temperature, it approaches a finite limit $\mathcal{F}_{1}=3 k_{B} T \Xi$ at $M \rightarrow 1$ where it should vanish from the definition of Re-flows.

This signals a singular dependence of the Re-flow on temperature. This singular dependence is not divergent at $M>2$. However, it becomes a real divergence in the limit of $M \rightarrow 1$, that is, the divergence of fourth-order contribution to the flow of von Neumann entropy. Indeed, already from the general expression (26), we deduce that

$$
\frac{d S}{d t}=\sum_{a, b}\left|A_{a b}\right|^{2} \delta\left(E_{a}-E_{b}\right) \frac{1}{p_{a}},
$$

that is, the states with lesser probabilities $p_{a}$ contribute most to the entropy flow! For our Fermi gas example, the entropy flow reduces to

$$
\frac{d S}{d t}=\Xi \int d E \frac{\left(f_{A}-f_{B}\right)^{2}}{f_{A} \tilde{f}_{A}} .
$$

For equal temperatures in the systems $A$ and $B$, this gives

$$
\frac{d S}{d t}=4 k_{B} T \Xi \sinh ^{2} \frac{e V}{2 k_{B} T} .
$$

The fourth-order contribution to the entropy flow thus diverges exponentially upon $T \rightarrow 0$.

We stress that this does not imply a divergence of the entropy flow in the limit of vanishing temperature; rather, it implies a singular, nonanalytical dependence on the coupling strength in this limit. To illustrate with a concrete example, let us turn to Eq. (1). The transmission coefficient $T_{0}$ plays here the role of the coupling strength. At $T=0$, the equation gives an evidently nonanalytical dependence $T_{0} \ln \left(T_{0}\right)$ that can not be expanded in Taylor series. Such expansion should, however, hold at any nonvanishing temperatures,

$$
\frac{d S}{d t} \frac{d t}{=} \sum_{n} A_{n}(T) T_{0}^{n}
$$

The only way to reconcile this with the nonanalytical dependence on $T_{0}$ is to conclude that $A_{n}(T)$ diverge in the limit of $T \rightarrow 0$. Since $T_{0}$ is proportional to the square of transmission amplitudes, the results of this paper give $A_{1}$ (second order) and $A_{2}$ (fourth order). Indeed, we have obtained that $A_{1}(T) \propto T^{-1}, A_{2} \propto T \exp \left(e V / k_{B} T\right)$, that is, diverge. The methods of Refs. 10 and 13 can, in principle, be applied to evaluation of temperature-dependent entropy flow, however, they have not been yet elaborated until the level that allows explicit evaluation of the coefficients in the above relation. The author is sure that the further elaboration of the results of Refs. 10 and 13 will confirm the perturbative calculations presented in this paper.

\section{CONCLUSIONS}

In this paper, we have shown that the flows of Rényi entropies are well defined for common setups encompassing a junction between two reservoirs $A$ and $B$. They are similar to flows of physical conserved quantities such as energy and charge. They vanish in thermal equilibrium and can be induced by the difference of chemical potentials and temperatures in the reservoirs. The flows characterize (quantum) information transfer between the reservoirs and generalize the flow of von Neumann entropy.

We develop a general quantum perturbation technique to compute these flows; this involves a set of parallel worlds. We consider second- and fourth-order terms. In the secondorder approximation, the flows can be related to golden rule transition events. As such, they satisfy a set of intuitive relations expressed by Eqs. (2), (24), and (20).

Our analysis of fourth-order corrections reveals interesting physics: the intuitive relations appear to be wrong, and the orders of perturbation theory exhibit divergences in the limit of low temperatures manifesting an intriguing nonanalytical dependence of the flows on coupling strength in the limit of weak couplings and vanishing temperatures.

\section{ACKNOWLEDGMENT}

The author is indebted to C. W. J. Beenakker, L. S. Levitov, K. Le Hur, I. Klich, and F. W. J Hekking for numerous important discussions.
${ }^{1}$ L. Amico, R. Fazio, A. Osterloh, and V. Vedral, Rev. Mod. Phys. 80, 517 (2008).

${ }^{2}$ R. Thomale, D. P. Arovas, and B. A. Bernevig, Phys. Rev. Lett. 105, 116805 (2010).

${ }^{3}$ P. Calabrese and J. Cardy, J. Stat. Mech. (2004) P06002.

${ }^{4}$ E. Fradkin and J. E. Moore, Phys. Rev. Lett. 97, 050404 (2006).

${ }^{5}$ M. Cramer, J. Eisert, M. B. Plenio, and J. Dreissig, Phys. Rev. A 73, 012309 (2006).
${ }^{6}$ H. F. Song, N. Laflorencie, S. Rachel, and K. Le Hur, Phys. Rev. B 83, 224410 (2011).

${ }^{7}$ A. Rényi, On Measures of Information and Entropy, Proceedings of the 4th Berkeley Symposium on Mathematics, Statistics and Probability, Vol. 1 (Univ. of Calif. Press, Berkeley, Calif., 1961), p. 547.

${ }^{8}$ This differs from the popular definition $\mathcal{S}_{M}=\ln \left[\operatorname{Tr}\left\{\hat{R}^{M}\right\}\right] /(M-$ $1)$, which would be extremely inconvenient to use in this paper. 
${ }^{9}$ M. Fagotti and P. Calabrese, Phys. Rev. A 78, 010306(R) (2008).

${ }^{10}$ C. W. J. Beenakker, Proceedings of the International School of Physics Enrico Fermi, Vol. 162 (IOS, Amsterdam, 2006) and references therein.

${ }^{11}$ L. S. Levitov, H. W. Lee, and G. B. Lesovik, J. Math. Phys. 37, 4845 (1996).

${ }^{12}$ I. Klich and L. Levitov, Phys. Rev. Lett. 102, 100502 (2009).

${ }^{13}$ H. F. Song, C. Flindt, S. Rachel, I. Klich, and K. Le Hur, Phys. Rev. B 83, 161408(R) (2011). The preprint version arXiv:1008.5191 [cond-mat] of this paper also outlines some results concerning Rényi entropies that are to be published.

${ }^{14}$ J. Cardy, Phys. Rev. Lett. 106, 150404 (2011).
${ }^{15}$ Yu. L. Klimontovich, Chaos Solitons Fractals 5, 1985 (1995).

${ }^{16}$ Yuli V. Nazarov and Y. Blanter, Quantum Transport: Introduction to Nanoscience (Cambridge University Press, Cambridge, 2009).

${ }^{17}$ I. Zutic, J. Fabian, and S. Das Sarma, Rev. Mod. Phys. 76, 323 (2004).

${ }^{18}$ Although this does not apply to spin currents, if the junction is characterized by some spin structure, spin currents do not have to be the same on both sides of the junction. See, e.g., A. Brataas, Y. V. Nazarov, and G. E. W. Bauer, Phys. Rev. Lett. 84, 2481 (2000); Yuli V. Nazarov, New J. Phys. 9, 352 (2007).

${ }^{19}$ L. D. Landau and E. M. Lifschiz, Quantum Mechanics (Butterworth-Heinemann, Oxford, 1998). 